\title{
The Application of Chaos Immune Genetic Algorithm in Sequencing Problem of Sports Meeting
}

\author{
Pan Ying \\ Harbin University, Engineering Institute Harbin \\ e-mail: py_0613@sina.com
}

\begin{abstract}
The sequencing of sports meeting is quite complicated due to the huge number of people and events. A new immune genetic algorithm aiming at solving the sports meeting sequencing problem is proposed in this paper, namely the chaos immune genetic algorithm, which combines the immune algorithm, chaos algorithm and genetic algorithm together. It generates the initial population by ergodic property and randomness of chaotic motion to speed up search, and adds new chaotic sequence by concentration calculation and adjustment of immune principle to supplement populations and increase population diversity so as to avoid trapping in local optimum. Local optimization is conducted after crossover and mutation by chaos near the optimal solution to improve the accuracy of solution. Experimental results show that this algorithm can quickly find the optimal solution and better accomplish its application in sequencing problem of sports meeting.
\end{abstract}

Keywords: Sequencing, Traveling salesman, Chaos, Genetic algorithm, Immune algorithm

\section{INTRODUCTION}

In recent years, most sports meetings in universities, middle and primary schools were manually sequenced, which were complicated and easy to go wrong ${ }^{[1]}$. Besides, it was unable to achieve good effects. The sequencing problem of sports meeting has been shown to be a difficult problem of $\mathrm{NP}$ as early as in the early $1960 \mathrm{~s}^{[2]}$. Sequencing algorithms that are currently used include heuristic search ${ }^{[3]}$, network optimization algorithm ${ }^{[4]}$ and genetic algorithm, etc. These algorithms can basically solve simple sequencing problems from the perspective of optimization, but there are still lots of problems in practical application that need to be further improved. A new immune genetic algorithm aiming at solving the sports meeting sequencing problem is proposed in this paper, namely the chaos immune genetic algorithm (NIGA). The chaos immune genetic algorithm reduces data redundancy in population and speeds up search by making use of ergodic property and randomness of chaotic motion. The basic idea is to take the objective function and the solution of the relevant problem in actual solving respectively as the antigen and the antibody of an immune system, and to conduct local optimization by the selfregulating mechanism of an immune system and chaos so as to quickly find the optimal solution or the approximate optimal solution. The immunological memory function and chaotic local optimization are able to speed up search and improve the overall searching ability, insuring a fast convergence to a globally optimal solution. It has the function of diversity preserving, being able to improve the global searching ability and avoid premature convergence. Therefore, it overcomes the "premature" problem that is hard to be solved in optimization process and ultimately obtains the global optimal solution.

\section{THE PRINCIPLE OF THE CHAOS IMMUNE GENETIC ALGORITHM}

Genetic algorithm(GA)is an effective probability searching algorithm based on natural selection and principles of genetics, conducting search and optimization by simulating biological evolution. It automatically acquires and accumulates knowledge of search space during searching process and adaptively control the searching process to obtain the optimal solution. This algorithm combines genetic algorithm, immune algorithm and chaos together, taking the problem to be solved as the antigen and the solution as the antibody. It describes the approximation level between the feasible solution and the optimal solution through the affinity of antigen and antibody. In resisting the intrusion of external antigens, the system produces antibodies through chaos and achieve self-adaption to the environment through judgment and adjustment of antibody concentration. It conducts local optimization by chaos near the optimal solution, improving the accuracy of solution and accelerating the speed of convergence. Concentration calculation of antibodies is one of the basic means of a system in maintaining the population diversity.

\section{A. The generation of the initial population}

Both immune algorithm and genetic algorithm initialize population randomly. Chaos is introduced in the algorithm of this paper ${ }^{[5]}$. Chaotic motion is characterized by ergodicity and randomness, so it is able to traverse all states without repetition in accordance with its own laws within a certain range. Therefore, if the initial population is generated by the principle of chaos, digital values will be uniformly distributed in the solution space, reducing possible data redundancy, providing a better solution at the beginning of iteration, and speeding up search. The well-known Logistic Equation is used in this paper to generate the initial population. The definition of the equation is:

$$
x_{n+1}=\mu x_{n}(1-x)
$$

In this equation, $x$ is a variable, $x \in[0,1], n=0,1,2 \ldots \ldots$. $\mathrm{u}$ is a control parameter. When $\mathrm{u} \in$ (3.56994], the mapping is in a chaotic state. In this algorithm, $\mathrm{n}$ represents the number of antibodies, $x_{n}$ stands for the $n^{\text {th }}$ antibody, $x_{n+1}$ stands for 
the $n+1^{\text {th }}$ antibody. The chaotic sequence space is $[0,1]$, so the state space can be also [0,1], which can be directly converted into the binary coded form.

\section{B. Differences and affinity of antibodies}

In order to effectively maintain and expand the diversity of individuals of population evolution, it is obliged to measure and evaluate differences between individuals. Apparently, the accuracy of difference measurement restricts the individual diversity of immune genetic algorithm. Here, differences between individuals are presented in the form of comentropy. Suppose the system consists of $\mathrm{N}$ antibodies of M genes, the comentropy $H_{j}(N)$ of the $j^{\text {th }}$ gene is:

$$
H_{J}(N)=\sum_{j=1}^{N}-p_{i, j} \log _{2} p_{i, j}
$$

In this equation, $\mathrm{p}_{\mathrm{ij}}$ is the allele of the $\mathrm{i}^{\text {th }}$ gene, originating from the probability of the $j^{\text {th }}$ gene.

The diversity of immune systems is calculated by the average comentropy:

$$
H(N)=\frac{\left|\sum_{j=1}^{M} H(N)\right|}{M}
$$

The affinity between antibody $\mathrm{V}$ and antibody $\mathrm{W}$ is defined as:

$$
a y_{v, w}=\frac{1}{1+H(2)}
$$

In this equation, $\mathrm{H}(2)$ represents the comentropy of antibody $\mathrm{V}$ and antibody $\mathrm{W}$. The value of $\mathrm{ay}_{\mathrm{v}, \mathrm{w}}$ ranges between $0 \sim 1$.

The affinity between antibody and antigen, namely the fitness, can be defined as:

$$
a x_{v}=\frac{1}{1+o p t_{v}}
$$

In the above equation, opt $t_{v}$ stands for the matching degree of the antigen and the antibody $\mathrm{V}$. The value of $\mathrm{ax}_{\mathrm{V}}$ ranges between $0 \sim 1 . \mathrm{ax}_{\mathrm{v}}=1$ represents that the antigen and the antibody matches perfectly, which means that the antibody is the optimal solution.

\section{The calculation of diversity}

Comentropy is used in the system for defining the diversity of populations. In calculations of fitness and affinity:

$$
H(N)=\frac{\left|\sum_{j=1}^{M} H(N)\right|}{M}
$$

It can be known from the definition of comentropy that the larger the $H(N)$, the better the diversity of populations. In the initial stage of evolution, the population information is quite rich. The diversity of populations will decrease with the continuous improvement of evolution generations and fitness. The diversity is 0 when individuals in a population become consistent.

\section{The judgement of diversity}

The diversity threshold value of each generation is set as:

$$
Q(t)=A(\log |S|) e^{-\frac{B t}{G}}
$$

In this equation, $0<\mathrm{A}<1$. A is the adjustment coefficient, $\mathrm{B}>0$ is the accelerating factor, $\mathrm{t}$ is the number of generations of current evolution, $|\mathrm{S}|$ is the coded symbol set used by antibody, and $G$ is the total number of generations of evolution. In the evolutionary process, if the diversity populations is less than the threshold value, measures should be taken to improve the diversity.

\section{E. Local optimization near the maximum}

After crossover and mutation, sequence the affinity between antibodies and antigens of new populations so as to find out the optimal solution. Convert the binary field corresponded to the optimal solution into the primary problem field $x$, and conduct chaotic iteration according to the equation (1)in order to find a favorable value replacing $Z$. And then convert it back to the binary field.

\section{F. Basic steps and flow chart of the algorithm}

(1)Antigen input and parameter setting. The objective function is taken as the antigen input. Set the population size, the crossover probability, and the mutation probability.

(2)The generation of initial antibodies. Take variables as antibodies and generate $N$ individuals by the logistic mapping so as to form the initial population.

(3)Calculation of affinity and concentration.

(4)The judgement of terminal condition. If the current population contains the best individual, the algorithm stops and output the result. Otherwise, the algorithm continues.

(5)The calculation and judgement of diversity. If the diversity of population is less than the threshold value, measures should be taken to improve the population diversity.

(6)The adjustment of diversity. Generate $m$ individuals by the logistic mapping and replace $m$ individuals randomly selected from the evolutionary population until the diversity exceeds the threshold value.

(7)Conduct crossover and mutation operations to the current population so as to generate a new population.

(8)Sequence the affinity between antigens and antibodies of the new population to find out the optimal solution and conduct local optimization near the optimal solution.

(9)Population updating.

The flow chart of the algorithm is shown as below. 


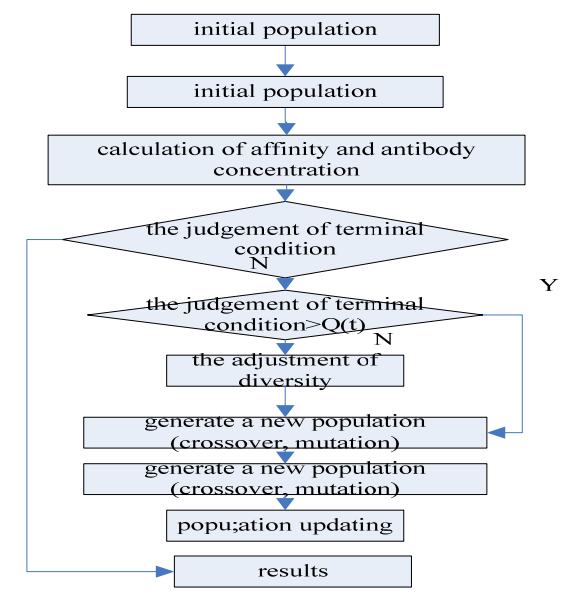

\section{THE MATHEMATICAL MODEL OF SPORTS MEETING SEQUENCING}

The sequencing of events of a small sports meeting is taken as an example of finding solutions by chaos immune genetic algorithm. Table 1 is the registration form of the small sport meeting. There are 40 athletes taking part in 14 events. The first line represents the 14 sports events and the first column represents 40 athletes. "\#” in the table indicates that athletes take part in the event. For Table 1, it is required to arrange sports events reasonably so that the number of athletes participating in two events in a row can be as small as possible.

\section{1)Cost matrix}

Substitute \# in the registration form with 1 and put 0 into all blanks so as to form the matrix a that is composed of 0 and 1 . And then combine the $i^{\text {th }}$ column and the $j^{\text {th }}$ column of the matrix with MATLAB software ${ }^{[6,7]}$, forming a matrix $x[i][j]$. The result from the combination of the $\mathrm{j}^{\text {th }}$ column and the $\mathrm{i}^{\text {th }}$ column is the same as the one from the combination of the $i^{\text {th }}$ column and the $j^{\text {th }}$ column, so $a[j][i]=a[i][j]$. Each column appears only once, so there will not be combinations of the same column. When $\mathrm{i}=\mathrm{j}, \mathrm{a}[\mathrm{i}][\mathrm{j}]=0$. It also needs to be noted here: no combination of the same column is designed in MATLAB and only the calculation method of combination between two lines is designed. So matrix a is transposed and represented by matrix $b$. The matrix $x[i][j]$, namely the cost matrix, is obtained by processing matrix $b$ and combining matrix a. Specific procedures omitted.

2)Establish the 0-1 integral linear programming mathematical model of sports events sequencing problem based on the inspiration of TSP problem.

$$
\begin{cases}\min & z=\sum_{\substack{i, j=1 \\ i \neq j}}^{n} c_{i j} x_{i j} \\ \text { s.t. } & \sum_{\substack{i=1 \\ n}}^{n} x_{i j}=1, \quad j=1,2, \cdots, n \\ & \sum_{j=1}^{n} x_{i j}=1, \quad i=1,2, \cdots, n \\ & u_{i}-u_{j}+n x_{i j} \leq n-1, \quad 2 \leq i \neq j \leq n \\ & x_{i j}=0,1, \quad i, j=1,2, \cdots, n \\ & u_{i} \geq 0, \quad i=2,3, \cdots, n\end{cases}
$$

\section{RESUltS ANALYSIS}

The optimal sequence can be obtained through the process executions. ABCDEFJHIGKLMN represents respectively the first event to the fourteenth event. Choose the smallest total loop overhead. It can be known from the results that the smallest total loop overhead is 2, so the sequence of events that optimizes th objective function goes like this:

8151110362941371214 or 141226371151 8941310 or 2637115189413101214 or 141210 1349815711362

In reality, there are four possibilities of sports events sequence when the number of athletes who participate in two events in a row needs to be as small as possible. It is able to make arbitrary selection. Therefore, in accordance with the above-mentioned sequence, there are only two athletes taking part in two events in a row.

\section{CONCLUSION}

The chaos immune genetic algorithm introduced in sports events sequencing means that the space of the problem space and the relevant solution space are parallel to antigen and antibody, and the reproduction of the local optimal is controlled by concentration control ${ }^{[13]}$. On this basis, the searching is accelerated by introducing chaos into the initial population and diversity adjustment. This project is supported by the educational scientific research project of the "12th Five-Year Plan" of the higher education association in Heilongjiang Province. No: HGJXH B2110958.

\section{REFERENCES}

[1] Liu Gong, Zhang Ping. The Sequencing Process of Small and Medium-sized Track and Field Games[J]. Journal of Gansu Normal Colleges. 2001,6(5):76 77.

[2] Lin Feng, Yang Jinyuan. Track and Field Sports Meeting Scheduling based on Genetic Algorithm[J]. Journal of Jilin Institute of Chemical Technology. 2010,6.

[3] Li Dan, Wang Lichen. An Algorithm Model of Popular Track and Field Sports Meeting Schedule[J]. Journal of Physical Education Institute of Shanxi Teachers University, 2002,17(4):55 57.

[4] Zhang Jianxun, Liu Minghua. The Static State Model and Algorithm for the Automatic Arrangement of the Competition Schedule of a Track and Field Sports Meet[J]. Journal of Lanzhou Railway Institute, 1994,14(2):86 92.

[5] Wu Xiangxing, Chen Zhong. An Introduction of Chaos. Shanghai Science and Technology Press. 1997. 
[6] He Renbing. Engineering Computing and Application of MATLAB 6[M]. Chongqing University Press, 2001,12.
[7] Yan Lin. Mathematics and Experiments-Applications of MATLAB and QBASIC. Science Press, 2005, 9.

Table 1 . The registration form of a small sport meeting

\begin{tabular}{|c|c|c|c|c|c|c|c|c|c|c|c|c|c|c|}
\hline $\begin{array}{c}\text { events } \\
\text { athletes }\end{array}$ & 1 & 2 & 3 & 4 & 5 & 6 & 7 & 8 & 9 & 10 & 11 & 12 & 13 & 14 \\
\hline 1 & 0 , & 1 , & 1 , & 0 , & 0 , & 0 , & 0 , & 0 & 1 , & 0 , & 0 , & 0 , & 1 , & 0 \\
\hline 2 & 0 , & 0 , & 0 , & 0 , & 0 , & 0 & 0 , & 1 , & 0 , & 0 , & 1 , & 1 , & 0 , & 0 \\
\hline 3 & 0 , & 1, & 0 , & 1 , & 0 , & 0 & 0 & 0 & 0 , & 1 , & 0 , & 0 & 0 , & 0 \\
\hline 4 & 0 , & 0 , & 1 , & 0 , & 0 , & 0 , & 0 , & 1 , & 0 , & 0 , & 0 , & 1 , & 0 , & 0 \\
\hline 5 & 0 & 0 & 0 & 0 & 0 & 0 & 0 , & 0 & 0 , & 0 & 1 , & 0 , & 1, & 1 \\
\hline 6 & 0 , & 0 , & 0 & 0 , & 1 , & 1 , & 0 , & 0 & 0 , & 0 , & 0 , & 0 , & 0 , & 0 \\
\hline 7 & 0 , & 0 , & 0 , & 0 , & 0 , & 0 , & 0 , & 0 & 0 , & 0 , & 0 , & 1 , & 1 , & 0 \\
\hline 8 & 0 , & 0 , & 0 , & 0 , & 0 , & 0 , & 0 , & 0 & 0 , & 1 , & 0 , & 0 , & 0 , & 1 \\
\hline 9 & 0 , & 1 , & 0 & 1 , & 0 , & 0 , & 0 , & 0 , & 0 , & 1 , & 1 , & 0 , & 0 , & 0 \\
\hline 10 & 1 , & 1 , & 0 & 1 , & 0 , & 0 , & 1 , & 0 , & 0 , & 0 , & 0 , & 0 , & 0 , & 0 \\
\hline 11 & 0 , & 1 , & 0 & 1 , & 0 , & 0 , & 0 , & 0 , & 0 , & 0 , & 0 , & 0 , & 1 , & 1 \\
\hline 12 & 0 , & 0 , & 0 & 0 , & 0 , & 0 & 0 & 1 , & 0 , & 1 , & 0 , & 0 & 0 , & 0 \\
\hline 13 & 0 & 0 , & 0 , & 0 & 1 , & 0 & 0 & 0 & 0 , & 1 , & 0 , & 0 & 0 & 1 \\
\hline 14 & 0 , & 0 , & 1 , & 1 , & 0 , & 0 & 0 , & 1 , & 0 , & 0 , & 0 , & 0 , & 0 , & 0 \\
\hline 15 & 0 , & 0 , & 1 , & 0 , & 0 , & 0 & 0 , & 1 , & 0 , & 0 , & 0 , & 1 , & 0 & 0 \\
\hline 16 & 0 , & 0 , & 0 & 0 , & 0 , & 0 & 0 & 0 & 1 , & 0 , & 1 , & 1 , & 0 , & 0 \\
\hline 17 & 0 , & 0 , & 0 , & 0 , & 0 & 1, & 0 & 0 & 0 , & 0 & 0 , & 0 & 0 & 1 \\
\hline 18 & 0 , & 0 , & 0 , & 0 , & 0 , & 0 , & 1 , & 0 , & 0 , & 0 , & 0 , & 1 , & 0 , & 0 \\
\hline 19 & 0 & 0 , & 1 , & 0 , & 0 , & 0 & 0 , & 0 , & 0 , & 1 , & 0 , & 0 , & 0 & 0 \\
\hline 20 & 1, & 0 & 0 & 1, & 0 & 0 & 0 , & 0 & 0 , & 0 & 0 & 0 , & 0 & 0 \\
\hline 21 & 0 , & 0 , & 0 , & 0 , & 0 , & 0 & 0 , & 0 , & 1 , & 0 , & 0 , & 0 , & 0 , & 1 \\
\hline 22 & 0 , & 1 , & 0 , & 0 , & 1 , & 0 & 0 , & 0 , & 0 , & 0 , & 0 , & 0 & 0 , & 0 \\
\hline 23 & 0 , & 0 & 0 , & 0 , & 0 , & 0 & 1, & 0 , & 0 , & 0 , & 0 , & 1, & 0 , & 0 \\
\hline 24 & 0 , & 0 , & 0 , & 0 , & 0 , & 0 , & 1 , & 1 , & 0 , & 0 , & 0 , & 0 & 1 , & 1 \\
\hline 25 & 1, & 1, & 0 , & 0 , & 0 , & 0 , & 0 , & 0 , & 0 , & 1, & 0 , & 0 , & 0 , & 0 \\
\hline 26 & 0 , & 0 , & 0 , & 0 , & 1 , & 0 & 0 , & 0 & 0 , & 0 , & 0 , & 0 & 0 , & 1 \\
\hline 27 & 0 , & 0 & 0 , & 0 , & 0 & 1, & 0 & 0 , & 0 , & 0 & 1, & 0 & 0 , & 0 \\
\hline 28 & 0 , & 1, & 0 , & 0 , & 0 , & 0 , & 0 , & 1 , & 0 , & 0 , & 0 , & 0 & 0 , & 0 \\
\hline 29 & 1, & 0 , & 0 , & 0 , & 0 , & 0 & 0 & 0 , & 0 , & 0 , & 1, & 1, & 0 , & 0 \\
\hline 30 & 0 , & 0 , & 0 , & 1 , & 1 , & 0 & 0 , & 0 & 0 , & 0 , & 0 , & 0 , & 0 & 0 \\
\hline 31 & 0 , & 0 , & 0 & 0 , & 0 , & 1 , & 0 & 1 , & 0 , & 0 , & 0 , & 1 , & 0 , & 0 \\
\hline 32 & 0 , & 0 & 0 , & 0 , & 0 & 0 & 1, & 0 & 0 , & 1, & 0 , & 0 & 0 & 0 \\
\hline 33 & 0 , & 0 , & 0 , & 1 , & 0 , & 1 , & 0 , & 0 , & 0 , & 0 , & 0 , & 0 , & 0 , & 0 \\
\hline 34 & 1, & 0 & 1 , & 0 & 0 , & 0 & 0 & 0 & 0 , & 0 & 0 & 0 & 1 , & 1 \\
\hline 35 & 0 , & 0 , & 0 & 0 , & 1, & 1 , & 0 & 0 & 0 , & 0 , & 0 , & 1 , & 0 , & 0 \\
\hline 36 & 0 & 0 & 0 , & 1 , & 0 & 0 & 1, & 0 & 0 , & 0 & 0 , & 0 & 0 & 0 \\
\hline 37 & 1 , & 0 & 0 , & 0 , & 0 , & 0 , & 0 , & 0 , & 1 , & 1 , & 0 , & 0 & 0 , & 0 \\
\hline 38 & 0 , & 0 , & 0 & 0 , & 0 , & 1 , & 0 & 1, & 0 , & 1, & 0 & 0 , & 0 & 1 \\
\hline 39 & 0 , & 0 , & 0 , & 0 , & 1, & 0 , & 0 , & 1 , & 1 , & 0 , & 0 , & 0 & 1 , & 0 \\
\hline 40 & 0 , & 0 , & 0 , & 0 , & 0 , & 1 , & 1 , & 0 , & 1 , & 0 , & 0 , & 0 , & 1 , & 0 \\
\hline
\end{tabular}

